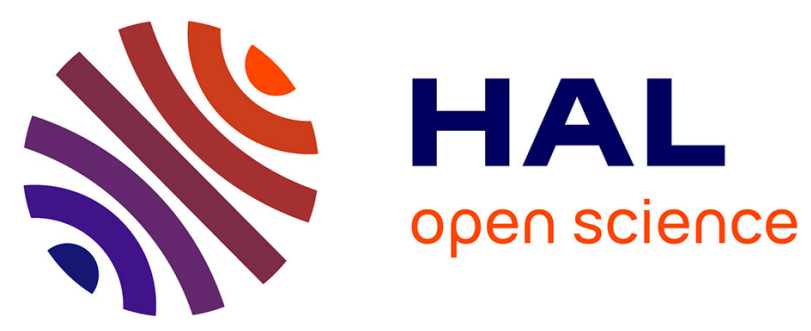

\title{
Optimal energy consumption algorithm based on speed reference generation for urban electric vehicles
}

Carlos Flores, Vicente Milanés, Joshué Pérez, David González, Fawzi

Nashashibi

\section{- To cite this version:}

Carlos Flores, Vicente Milanés, Joshué Pérez, David González, Fawzi Nashashibi. Optimal energy consumption algorithm based on speed reference generation for urban electric vehicles. IV2015 2015 IEEE Intelligent Vehicles Symposium, IEEE, Jun 2015, Seoul, South Korea. pp.730 - 735 10.1109/IVS.2015.7225771 . hal-01251040

\author{
HAL Id: hal-01251040 \\ https://hal.inria.fr/hal-01251040
}

Submitted on 5 Jan 2016

HAL is a multi-disciplinary open access archive for the deposit and dissemination of scientific research documents, whether they are published or not. The documents may come from teaching and research institutions in France or abroad, or from public or private research centers.
L'archive ouverte pluridisciplinaire HAL, est destinée au dépôt et à la diffusion de documents scientifiques de niveau recherche, publiés ou non, émanant des établissements d'enseignement et de recherche français ou étrangers, des laboratoires publics ou privés. 


\title{
Optimal Energy Consumption Algorithm based on Speed Reference Generation for Urban Electric Vehicles
}

\author{
Carlos Flores, Vicente Milanés, Joshué Pérez, David González, Fawzi Nashashibi
}

\begin{abstract}
Power consumption and battery life are two of the key aspect when it comes to improve electric transportation systems autonomy. This paper describes the design, development and implementation of a speed profile generation based on the calculation of the optimal energy consumption for electric Cybercar vehicles for each of the stretches that are covering. The proposed system considers a commuter daily route that is already known. It divides the pre-defined route into segments according to the road slope and stretch length, generating the proper speed reference. The developed system was tested on an experimental electric platform at Inria's facilities, showing a significant improvement in terms of energy consumption for a pre-defined route.
\end{abstract}

\section{INTRODUCTION}

Electric vehicles (EVs) are getting more and more attention because their contribution toward eco-friendly cities. Non-emission vehicles will definitely help to improve citizen's daily life, reducing dramatically both noise and pollution [1]. For this reason, some governments-i.e. Canada [2]-have carried out studies to figure out the emissions according to the kind of vehicle. Results showed that light duty gas vehicles are the biggest producer of $\mathrm{CO}_{2}$ and the second greatest producer of $\mathrm{N}_{2} \mathrm{O}$ and methane, which makes them the main contributors towards gas emissions-because of their higher market penetration. In United States [3], the transportation sector consumes three-quarters of the total burned petroleum, which makes it the second largest carbon emitter in the country. Because of this, electric vehicles are an adequate solution to reduce greenhouse gas (GHG) emissions and pollution produced by road transport systems.

On the other hand, EVs present serious limitations for their market deployment. Specifically, there are two unsolved challenges: 1) battery charge [4]: how long it takes to fully load when running off at driving; and 2) battery life [5]: how much energy will last when driving. Recent years have shown a lot of development on battery technologiesi.e. the mixed structures using supercapacitors [6] or fuel cells [7]. However, EVs autonomy remains considerably lower than gas-powered vehicles. This paper deals with this second challenge, proposing an intelligent modular algorithm that provides better performance in order to improve EVs autonomy.

Specifically related with the solution to the EVs autonomy problem, there are two main ways for improving battery

Authors are with the Robotics and Intelligent Transportation Systems (RITS) Team, Inria Paris-Rocquencourt, Domaine de Voluceau, 78153 Le Chesnay, France \{carlos-eduardo.flores-pino, vicente.milanes, joshue.perez_rastelli, david.gonzalez-bautista, fawzi.nashashibi\} dinria.fr life. One of them is optimizing the technology available in the car such as better batteries or more efficient motor and mechanical parts. This would bring better results when trying to extend batteries' life, but modifying the vehicle physical characteristics would mean increase the cost and technology, which is sometimes a very complex task. The other way for improving EVs autonomy is modifying the driving performance, considering all the circumstances around the vehicle to take advantage of them and performing a more efficient driving.

For the development of a reliable algorithm that takes into account the driving environment for extending battery life, several parameters have to be considered: distance, traffic state, traversing intersections and merging zones [8]. Having this in mind, optimal route generation algorithms are a suitable candidate to solve this problem. These methods offer a route, avoiding heavy traffic on urban roads and traffic lights in intersections [9], which would cause more unnecessary braking maneuvers. Nonetheless, none of those considerations really provides a reliable solution when trying to save battery life.

An optimal route planning would lead to a less energy consumption [10], where a real time state of the vehicle and the road layout are considered in order to make a speed profile plan. Once this optimal route is obtained from an algorithm-i.e. [11] and [12]-and the environment conditions are established, it is necessary to optimize the energy consumption for this pre-defined route. Drivers are usually covering the same trajectory from home to work everyday. However, they are usually following the traffic flow, including consecutive braking and accelerating maneuvers that significantly affect the battery life of EVs. The proposed method considers this regular situations in order to cover a pre-defined distance in the desired time but increasing the battery life as much as possible.

In the state-of-art of eco-driving, the optimal route algorithm is one of the main tools to improve vehicle's autonomy. [13] presents an approach that aims on finding the best route in terms of energy. It considers an origin, destination and the initial battery state of charge. With these parameters, the optimal route is computed by finding the path segments with the minimal energy cost. Even though this method offers a solution to extend the vehicle autonomy, it does not approach the problem from the point of view that this work assumes. This article presents an intelligent algorithm to generate a velocity profile that would allow a commuter go from one point to another taking a certain time, achieving the less energy consumption possible for electric vehicles. 
The rest of the paper is structured as follows. The problem formulation that motivated this research is described in section II. Section III presents the experimental platform and its adaptation for carrying out the proposed work. The speed profile algorithm is proposed in section IV. Experimental results-including a comparison with a human driver-are shown on section $\mathrm{V}$ and, finally, the conclusions derived from this work and possible future works are discussed.

\section{Problem Formulation}

This work assumes as starting point a commuter trip that everyday covers the same route from one point to another, spending the same time. We are also assuming that the vehicle is equipped with the proper sensor technology for allowing it to recognize his environment and where it is localized. It means that it will be able to autonomously drive-i.e., longitudinal control-which means less energy consumption through a more efficient way of driving. For doing so, some initial considerations were taking into account, as follow:

1) The route that will be covered by the commuter has been selected as the optimal trajectory, using an intelligent algorithm [14]. This route has been selected taking into account the distance to cover, the amount of intersections to pass through or how heavy the traffic is by the time that the route computation is requested, among other parameters.

2) The vehicle is traveling through a fluid traffic flow and, as a consequence, the precedent vehicle impact has not been taken into consideration on this first approach. More vehicle scenarios will be considered for future improvements.

3) In order to focus on the longitudinal control and speed profile generation, the possible obstacles that would be faced by the vehicle-i.e. pedestrians, cyclist or others cars-are not taken into account when the algorithm is executed. This approach is focused on a more efficient driving that comes after having a route selected. Real time control and interaction with other elements are proposed as future works.

With this premises, this work tries to find an intelligent and modular algorithm for improving the way that a selected route is covered in terms of efficiency. The unique requirement is that the trip duration has to be the same. Then, the goal is to avoid unnecessary power peaks as well as wasting more power than required on a certain situation.

\section{EXPERIMENTAL PLATFORM}

This section describes the experimental platform used for the validation on the real tests of the proposed algorithm. This platform is a four-wheeled robotized vehicle equipped with a perception system [15], using a SLAM (Simultaneous Localization and Mapping) technique for vehicle location. It allows the vehicle to compare the information perceived by the LIDAR (Light Detection and Ranging) with a preloaded map containing the route, deducing a match degree between them. Thanks to this, the vehicle is able to know which are both its position and its heading angle. The platform is also equipped with two lasers for obstacle detection. Two encoders are installed over the rear wheels and connected to the main computer through a Controller Area Network (CAN) interface. Figure 1 shows a picture of the electric Cybercar used.

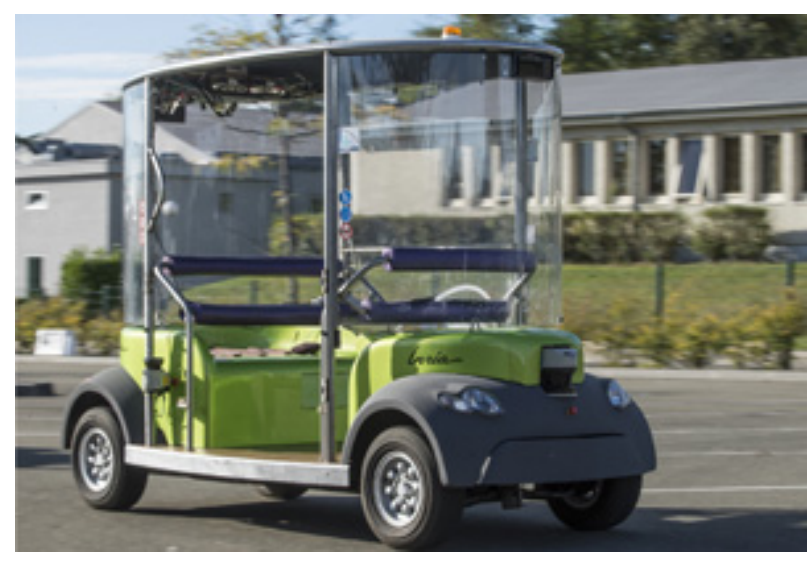

Fig. 1. Cybus vehicle, driving on INRIA's facilities

After the perception stage where the vehicle is located, the decision stage is responsible of the intelligence of the vehicle. In previous works, automated driving capabilities were developed to follow the predefined trajectory without any human intervention (see [16] and [17] for details). Decision stage is also able to dynamically change the route when an obstacle is detected or a lane-change is required. The control block manages the orders given at this stage, comparing them with the vehicle position in order to generate the final commands (lateral and longitudinal control) to the actuators (motor driver, steering wheel). This work aims on modifying the longitudinal control in order to improve the energy consumption taking into account the road layout.

\section{Speed Profile Algorithm}

This section describes the proposed algorithm for reducing the energy consumption of a vehicle from one point to another taking a specific time. This implemented algorithm considers the road layout that the vehicle will face on the selected route and the distance to cover. It also takes into account the vehicle dynamics to compute the driving losses using an energy model before starting the route.

\section{A. Algorithm Inputs}

The algorithm uses different information coming from the pre-defined route to be covered and the on-board sensor systems. All this information is received from the perception and decision stages. The inputs required by this speed profile algorithm are specified below:

1) Pre-loaded Route: In the same way that a commercial GPS works, it is necessary to have a preloaded file containing the points that describe the trajectory to be followed by the vehicle in a 3D map. The processing of this method is based on a pre-loaded data that have been added to the SLAM technique for localization. This control points are 
used to determine the different stretches to generate the proper reference speed in each of them. Altitude is used to compute the road layout, which is required in order to apply the energy model.

2) Vehicle Properties: Some of the vehicle characteristics are required for the energy model. In order to estimate the total energy needed by the vehicle to complete the route, a dynamic model is applied, which describes the amount of energy required to go on a certain speed, over a route with a specified slope and length. Equation 1 describes this model, where $M$ is the vehicle mass, $g$ is the gravitational acceleration, $\theta$ denotes the road slope, $v$ the vehicle speed, $A_{f}$ the vehicle front area, $C_{d}$ and $\mu_{r}$ the aerodynamic drag and rolling resistance coefficient respectively. As described on [13], equation 1 estimates the energy demand for each route segment taking into account both aerodynamic, gravitational and rolling resistance losses.

$E_{i}\left(\theta_{i}, d_{i}, v_{i}\right)=\frac{\rho}{2} \cdot A_{f} \cdot C_{d} \cdot v_{i}^{2} \cdot d_{i}+M \cdot g \cdot d_{i} \cdot\left(\mu_{r} \cdot \cos \theta_{i}+\sin \theta_{i}\right)$

3) Instant Power Consumed: For determining the power consumption on real time, an instant power measurement system was installed on the prototype. It allows to know how much power is demanding the vehicle on real time, permitting to check the behavior of the solution proposed. For the instant power measurement is required both the current and voltage from the batteries. For this reason, two modular systems were added to measure both variables. The first one is composed by a Hall Effect sensor biased by a \pm 12 volt power source and its main function is to translate the output current from the batteries to a low current output with a rate of $2000: 1$. Then, the current is conducted through a load whose voltage drop is measured by an I/O analog card connected to the PC via an USB port. For the voltage measurement a voltage divisor is implemented with a ground insulator on its output. The signal is acquired by the same card, being processed by the PC. Figure 2 shows the electronic scheme used for the installation of the current and voltage measurement systems.

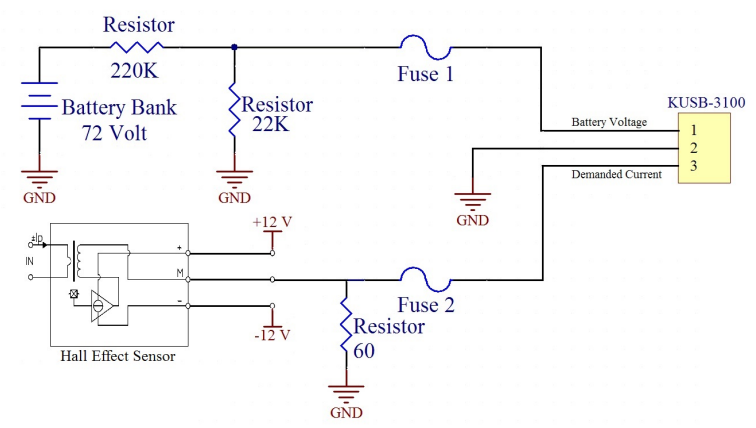

Fig. 2. Improvements required to the control architecture, acquisition and conditioning stage.

\section{B. Speed Profile Generation}

After loading the route selected by the commuter and the vehicle dynamic characteristics for the calculations, the next step is to divide the trajectory into segments-as shown on figure 3-with the same road slope. These route divisions have the same theoretical power consumption required. Once the division is set, the total energy required for the whole route is calculated using (equation 1) and adding every segment contribution to the total. Then, the whole energy estimated for completing the route divided by the time is determined. It brings as result the mean power that must be consumed all along the path, according to equation 2 . Therefore, the following conditions are satisfied: the time passed from the start until the end is the desired by the commuter and the power consumed through the whole travel is not greater than the required, so it is possible to get the best energy consumption rate.

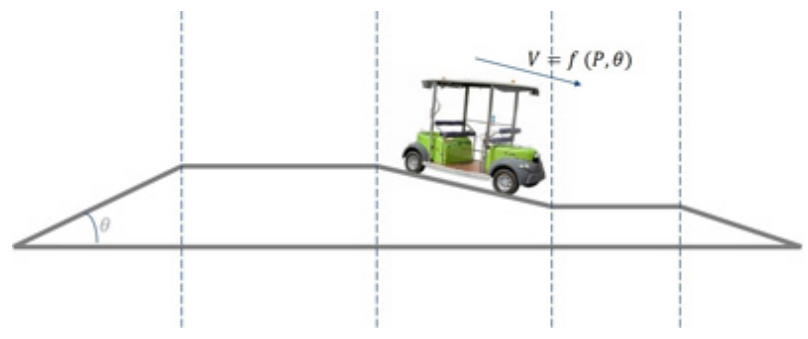

Fig. 3. Road splitting illustration, in order to apply the energetic model over each segment.

$$
\bar{P}=\sum_{i=0}^{n} \frac{E_{i}\left(\theta_{i}, d_{i}, v_{i}\right)}{t_{\text {total }}}
$$

In order to obtain a more regular speed profile, a weighing process based on the distance and the difference between the segment slope and the mean slope (equation 3) is applied, assigning to each segment the theoretical best power that should be consumed along it-see equation 4 . This consideration brings as result a greater power consumption assigned to upgrade segments and, on the other hand, downgrades are planned with less power cost. It produces a speed profile plan more stable with smaller differences among different layouts. This is also useful because makes the speed profile more likely as a common driver performance would do, bringing more comfort to the passenger.

$$
\begin{gathered}
\bar{\theta}=\sum_{i=0}^{n} \frac{\theta_{i} \cdot d_{i}}{d_{\text {total }}} \\
P_{i}=\bar{P} \cdot\left(1+k \cdot\left(\theta_{i}-\bar{\theta}\right)\right)
\end{gathered}
$$

Once each segment has its optimal power assigned, based on the road slope and the vehicle properties, the ideal speed is calculated by the employment of the energy model. This process sets the velocity profile for the entire route, completing it in the desired time. Figure 4 shows the complete algorithm flowchart, where each part is explained below. 
This flowchart describes the complete algorithm performance. First it starts reading the vehicle properties and the route characteristics, so it is able to apply the energetic model and estimate the optimal speed profile for the whole trajectory. It is also described how the vehicle generates the reference speed, once it has started the travel.

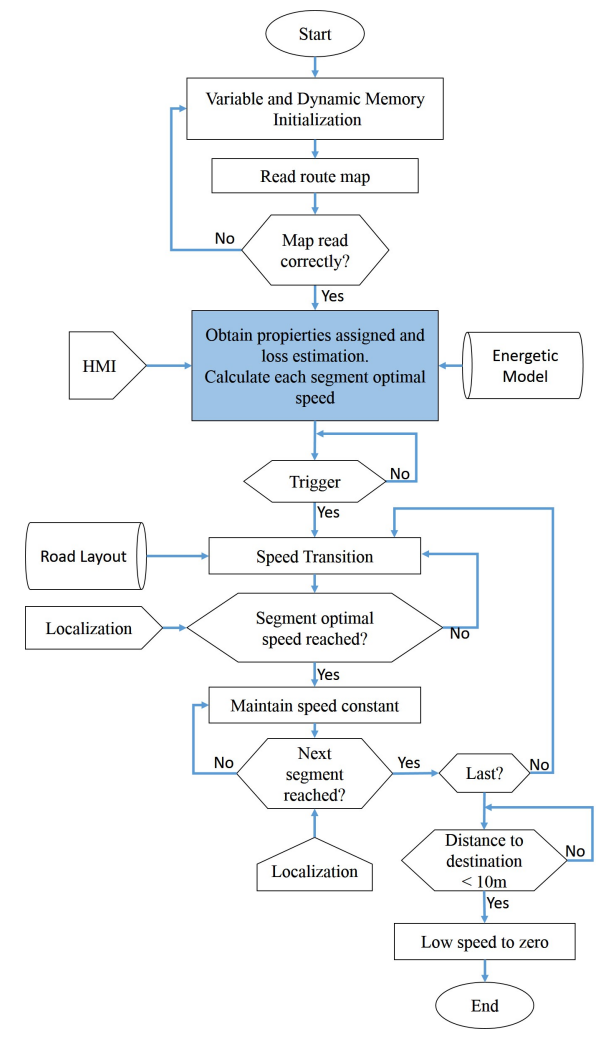

Fig. 4. Complete algorithm flowchart.

1) Dynamic Operation: Once the vehicle starts the route, the developed module is in charge of determining on which segment the vehicle is currently driving. Then, the optimal segment speed is generated according to the previously defined algorithm. When a new segment is detected, a smooth transition function is applied-see equation 5-to carry out an efficient velocity change.

$v_{i+1}=v_{i}+K \cdot t \cdot g \cdot\left[\cos \left(\frac{\pi}{2}-\Delta \theta\right)+\mu_{r} \cdot \sin \left(\frac{\pi}{2}-\Delta \theta\right)\right]$

\section{EXPERIMENTAL RESULTS}

This section presents the results obtained with the proposed algorithm using a pre-defined route. The experiments were carried out at Inria's facilities. The selected scenario includes upgrade, flat and downgrade segments, turning it into a good candidate for testing the performance of the proposed system. The figure 5 shows an image taken from Google Earth with the route selected (yellow line) and the elevation profile. The tests were done using a Cybus prototype, measuring the reference speed, the real speed and the instant power consumed by the vehicle on each travel. In order to have similar power conditions on each scenario, all the tests were made by employing fully-charged batteries.

\section{A. Comparative analysis}

Using the trajectory showed on figure 5 , two experiments are proposed to test the performance of the proposed system. The first scenario is the one explained on section II, where a commuter drives manually a vehicle, attempting to maintain a constant reference speed from a starting point to a destination, which is supposed to be a very efficient way to drive. The second scenario is when the intelligent speed profile algorithm is applied over the longitudinal control, acting over the energy consumption. The algorithm was tested multiple times for assuring its repeatability and because of this, one average and representative test was selected to be presented graphically in this work. The conditions in each scenario are the same, either the trajectory or the time established to cover the whole route. On each of the case graphics, the reference speed-red, the measured speed-blue-and the instant power consumed-green-are plotted.

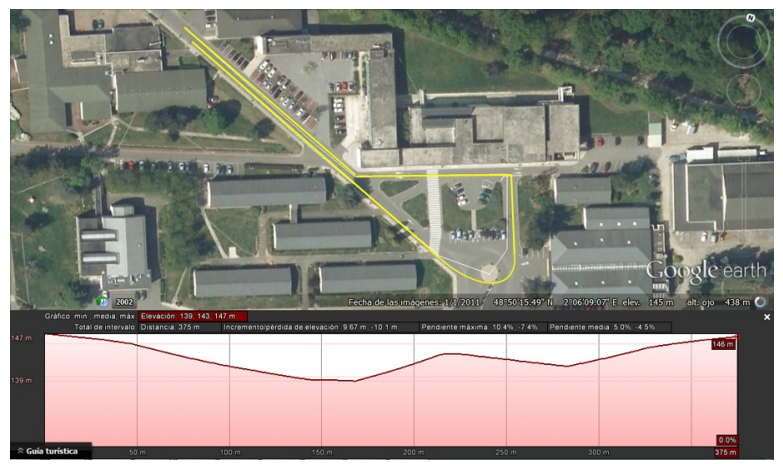

Fig. 5. INRIA's facilities with the route remarked on yellow.

1) Manual Driving: The figure 6 illustrates the reference speed generated by the driver-that is sent to the low level control, the measured speed and the instant power consumed by the vehicle. The power consumed results to be very irregular, leading to an inefficient driving way and causing higher energy consumption.

2) Speed Profile Algorithm: The route described on figure 5 is divided in segments in order to apply the Intelligent Speed Profile Algorithm (ISPA). Figure 7 shows how the reference speed changes along the trajectory, trying to pursuit the best energy consumption over each road layout. A good response from the energy consumption perspective is shown. However, there are some power peaks caused by the low level control on the longer downgrade segment.

3) Results Analysis: Figure 6 shows the performance of the vehicle while it is driven manually. The reference speed managed by the driver results irregular compared to the one generated by the automated system proposed. It means that the power consumed in the first case is higher since the speed 


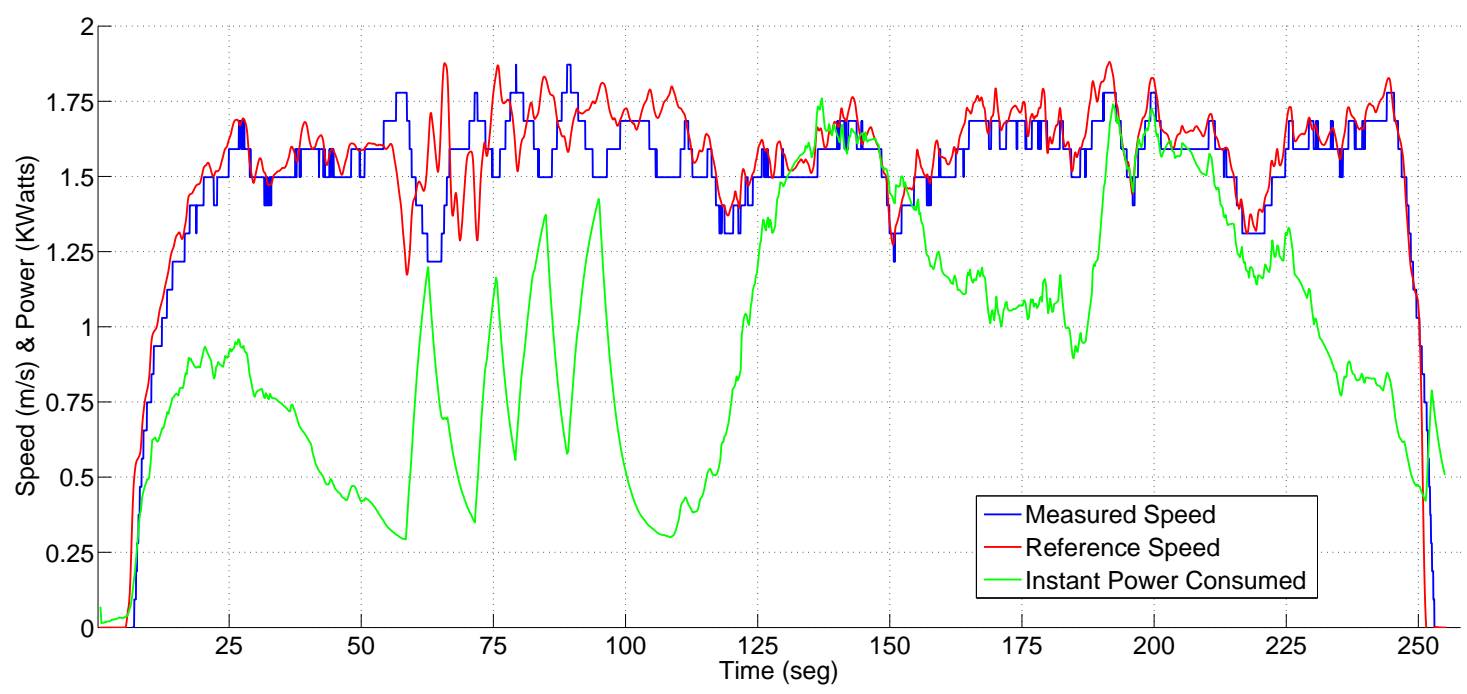

Fig. 6. Reference speed generated manually by the driver; and instant power consumed by the vehicle.

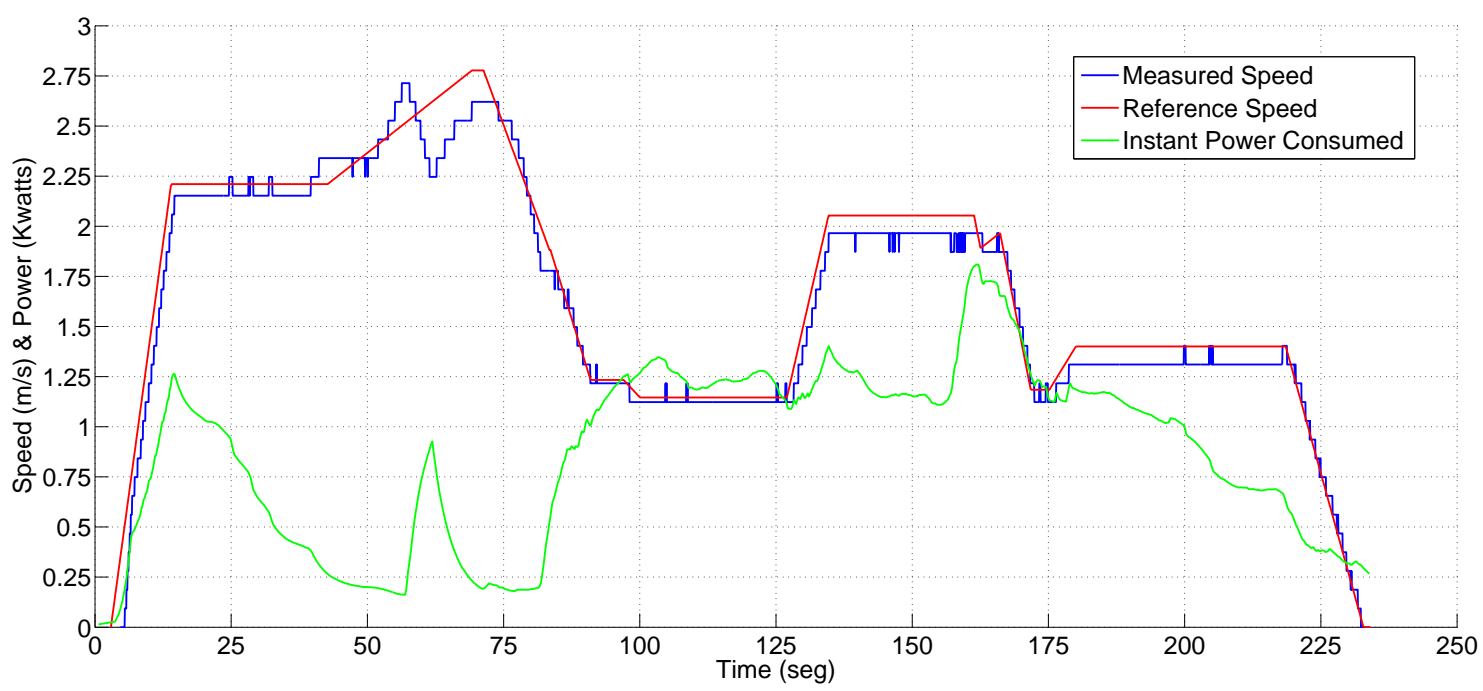

Fig. 7. Reference speed generated by the speed profile algorithm; and instant power consumed by the vehicle.

control is not efficient. On the other hand, the speed reference given by the ISPA is stable and does not oscillates as much as in the first case, which means that the energy management results even more efficient and proper to the road layout.

Table I shows the energy consumption for each of the routes. The ISPA algorithm saves $17.08 \%$ compared to the manual driver. The amount of energy consumed by the car was determined by integrating the instant power consumed while the vehicle was traveling along the route on each of the scenarios. The power peaks and the speed control problems observed on both figures (6 and 7) are due to the low level controller. The reason is that this experimental platform has been designed for applications which do not require a high precision speed control. Actuators and sensors were selected for this architecture in order to get a low cost platform.

\begin{tabular}{|c|c|c|}
\hline $\begin{array}{c}\text { Evaluation } \\
\text { Scenario }\end{array}$ & $\begin{array}{c}\text { Consumed Energy } \\
\text { (KJoules) }\end{array}$ & $\begin{array}{c}\text { Savings } \\
\text { Percentage (\%) }\end{array}$ \\
\hline Manual Driving & 243.23 & - \\
\hline ISP Algorithm & 201.68 & 17.08 \\
\hline
\end{tabular}

TABLE I

ENERGY CONSUMPTION FOR EACH SCENARIO, AND SAVING PERCENTAGE

\section{CONCLUSIONS AND Future Works}

This paper presents an algorithm for performing longitudinal vehicle control on an EVs with energy saving 
consideration. A solution to the electric vehicles autonomy problem is proposed by applying the concept of eco-driving and generating a reference speed that achieves big energy savings without changing any of the vehicle main resources (batteries, motor and power electronics).

This research proposes an intelligent algorithm able to generate a speed profile. It would make the vehicle cover a route in a more efficient way than a common driver would. The algorithm acts over a pre-defined trajectory and the time specified to cover it. The goal is to get the lower energy consumption in the fixed time.

As future work, traffic data will be introduced to modify the algorithm according to the traffic changes on real time. Additionally, higher speed ranges will be also considered. Finally, a high level system that modifies the speed reference in order to have a more efficient performance, solving the low level control problems will be investigated.

\section{ACKNOWLEDGMENTS}

Authors express their gratitude to the EU CityMobil-2 project and the RITS Team for its support in the development of this work.

\section{REFERENCES}

[1] Z. Cerovsky and P. Mindl, "Hybrid electric cars, combustion engine driven cars and their impact on environment," in Power Electronics, Electrical Drives, Automation and Motion, 2008. SPEEDAM 2008. International Symposium on, 2008, pp. 739-743.

[2] L. Graham, "Greenhouse gas emissions from light duty vehicles under a variety of driving conditions," in EIC Climate Change Technology, 2006 IEEE, 2006, pp. 1-8.

[3] H. Rakha, K. Ahn, and R. K. Kamalanathsharma, "Eco-vehicle speed control at signalized intersections using i2v communication," U.S. Department of Transportation, Tech. Rep., 2012.

[4] C. sheng Si, "Development research about the power battery management system of pure electric vehicle," in Consumer Electronics, Communications and Networks (CECNet), 2011 International Conference on, 2011, pp. 276-279.

[5] V. Marano, S. Onori, Y. Guezennec, G. Rizzoni, and N. Madella, "Lithium-ion batteries life estimation for plug-in hybrid electric vehicles," in Vehicle Power and Propulsion Conference, 2009. VPPC '09. IEEE, 2009, pp. 536-543.

[6] M. Tankari, M. Camara, and B. Dakyo, "DC-bus voltage control for multi-sources systems - battery and supercapacitors," in IECON 2011 37th Annual Conference on IEEE Industrial Electronics Society, 2011, pp. $1270-1275$.

[7] A. F. Burke, "Batteries and ultracapacitors for electric, hybrid, and fuel cell vehicles," Proceedings of the IEEE, vol. 95, no. 4, pp. 806-820, 2007.

[8] C. Li and S. Shimamoto, "A real time traffic light control scheme for reducing vehicles $\mathrm{CO} 2$ emissions," in Consumer Communications and Networking Conference (CCNC), 2011 IEEE, 2011, pp. 855-859.

[9] M. O. Rojas, J. P. Rastelli, N. Fawzi et al., "Fuzzy logic techniques for cybercars: a control and decision approach." in 22èmes rencontres francophones sur la Logique Floue et ses Applications (LFA 2013), 2013.

[10] D. Chrenko, A. Ravey, R. Roche, and D. Bouquain, "Autonomy estimation for EV based on road planning software," in Transportation Electrification Conference and Expo (ITEC), 2014 IEEE, 2014, pp. 14.

[11] M. Sachenbacher, M. Leucker, A. Artmeier, and J. Haselmayr, "Efficient energy-optimal routing for electric vehicles." in AAAI, 2011.

[12] V. Larsson, L. Johannesson, B. Egardt, and S. Karlsson, "Commuter route optimized energy management of hybrid electric vehicles," IEEE Transactions on Intelligent Transportation Systems, vol. 15, no. 3, pp. 1145-1154, 2014.
[13] T. Jurik, A. Cela, R. Hamouche, A. Reama, R. Natowicz, S. Niculescu, C. Villedieu, and D. Pachetau, "Energy optimal real-time navigation system: Application to a hybrid electrical vehicle," in Intelligent Transportation Systems - (ITSC), 2013 16th International IEEE Conference on, 2013, pp. 1947-1952.

[14] T. Jurik, A. Cela, R. Hamouche, R. Natowicz, A. Reama, S.-I Niculescu, and J. Julien, "Energy optimal real-time navigation system," IEEE Intelligent Transportation Systems Magazine, vol. 6, no. 3, pp. 66-79, 2014.

[15] T. Xia, M. Yang, R. Yang, and C. Wang, "Cyberc3: A prototype cybernetic transportation system for urban applications," IEEE Transactions on Intelligent Transportation Systems, vol. 11, no. 1, pp. 142-152, 2010.

[16] J. Rastelli, R. Lattarulo, and F. Nashashibi, "Dynamic trajectory generation using continuous-curvature algorithms for door to door assistance vehicles," in Intelligent Vehicles Symposium Proceedings, 2014 IEEE, 2014, pp. 510-515.

[17] D. Gonzalez and J. Perez, "Control architecture for cybernetic transportation systems in urban environments," in Intelligent Vehicles Symposium (IV), 2013 IEEE, 2013, pp. 1119-1124. 